\title{
Campylobacter enteritis presenting as an acute abdomen
}

\author{
D. J. HAY* \\ F.R.C.S.
}

\author{
Leela A. Ganguli $\dagger$ \\ M.B. B.S., Ph.D., Dp. BACT.
}

\author{
*University Department of Surgery, Manchester Royal Infirmary, and \\ †Department of Bacteriology, Hope Hospital, Salford
}

\begin{abstract}
Summary
A case is reported of a young patient with Campylobacter enteritis who underwent first appendicectomy and then a laparotomy because of the severity of the pain and the marked abdominal signs.

\section{Introduction}

There has been much recent interest in the Campylobacter group of organisms and many cases have been reported. A further case is described which presents a diagnostic problem.
\end{abstract}

\section{Case report}

A 19-year-old single girl was admitted with a 4-day history of cramping abdominal pain, nausea and greenish diarrhoea every 2 hr. On further questioning, she admitted to having been in close contact with a puppy which had diarrhoea. One year before she had had jaundice-diagnosed as chronic aggressive hepatitis, but this appeared to have remitted and she had been well for $>9$ months. On examination she was flushed and toxic with signs of local peritonitis in the right lower quadrant of the abdomen. It was felt that she probably had an enteritis but because of the markedly localized signs it was decided wisest to explore the abdomen. At operation through a gridiron incision the appendix was found to be normal but there was minimal thickening and inflammation of the caecum and terminal ileum but more proximally the small bowel appeared normal. The mesenteric lymph nodes were seen to be enlarged. The appendix was removed. For the next 3 days she was very well and the diarrhoea settled down.

On the 4th postoperative day the diarrhoea recurred although her general condition was satisfactory. The results of stool cultures done at admission showed a heavy growth of Campylobacter jejuni and so treatment with erythromycin stearate was commenced. However, her general condition suddenly deteriorated later the same day. The abdomen showed signs of generalized peritonitis. Plain abdominal radiology showed scattered small bowel fluid levels but no free gas. Nevertheless it was felt that the signs were so marked that further operation was mandatory.

At laparotomy there was an excess of clear fluid in the peritoneal cavity. The site of the appendicectomy was intact. The small bowel was grossly distended along its whole length. The upper jejunum and lower ileum although distended were otherwise normal but the middle half of the small bowel was dusky blue with multiple circumferential haemorrhagic patches which in some areas were diffuse. Despite the gross discoloration of the small bowel, the sheen of the serosa was not reduced and so it was felt that the damage was predominantly mucosal. Gross mesenteric lymphadenopathy was apparent. A Savage's decompressor was inserted through a small stab incision in the distal ileum and the small bowel decompressed. A drain was inserted to the region of the appendix stump in case the manipulation had disturbed what had been, until then, a satisfactory closure and the abdomen was closed in layers. Her symptoms settled on intravenous feeding and the erythromycin stearate.

\section{Bacteriology}

Stool cultures from the patient after admission showed a growth of $C$. jejuni. After treatment with erythromycin stearate, the stool cultures became negative on the 3rd day after the second laparotomy. Stool cultures were obtained from the puppy from which she was believed to have contracted the infection and these showed a growth of the same organism. Positive cultures were also obtained from the stools of her boy friend who had diarrhoea and had been in contact with the animal. Serological testing and biotyping using strains and sera from both human cases and the dog showed that they were identical and confirmed that the dog was the source of infection. Paired sera from the patient showed an eightfold rise in titre to all strains.

\section{Discussion}

Campylobacter (Greek-'curved rod') jejuni is not a new organism. The type species, C. fetus, was formerly described as Vibrio fetus and was known to 
cause spontaneous abortion in cattle. The subspecies $C$. jejuni has been most commonly associated with human disease. Following the work of Skirrow (1977) it is now recognized as a cause of food poisoning in Britain. The rate of isolation in patients with diarrhoea has been $3-8 \%$ using special culture techniques including incubation at $42^{\circ} \mathrm{C}$.

The source of the infection is commonly undercooked poultry (Simmons and Gibbs, 1977; Skirrow, 1977). Dogs with diarrhoea have also been shown to be a source, possibly by consuming chicken droppings (Wheeler and Borchars, 1961 ; Blaser et al., 1978).

The clinical picture in children is usually that of a mild enteritis which lasts only a few days and spontaneously resolves with minimal constitutional upset (Wheeler and Borchars, 1961). In adults, the condition may be more severe with colicky abdominal pain as the most disabling symptom in addition to the diarrhoea which may be profuse, and sometimes bloodstained. Some patients are severely ill and laparotomy has been performed in a few patients because of the severity of the pain. Deaths have been described notably in the old or very young (King, 1962; Evans and Dadswell, 1967; Hallett, Botha and Logan, 1977). The findings described in these papers were similar to those found at operation on the present patient. In particular the mid-small gut is affected rather than the terminal ileum as described for typhoid enteritis.

In the present case, the source of infection, clinical presentation and course of the illness are typical of Campylobacter enteritis apart from the fact that this patient was more severely affected than most. I⿳⺈⿴囗十一 may be that she was infected with greater number of the infecting organism as the dog involved actually slept on her bed. A further predisposing factor might have been the previous chronic aggres sive hepatitis diagnosed one year before this episode?

Treatment with erythromycin stearate is noe contentious but the authors would suggest tha decompression of the small bowel in patients whळ come to laparotomy and in whom severe distensiore of the gut is found, is a helpful and perhaps lifesaving measure.

\section{Acknowledgments}

We thank Dr M. B. Skirrow, Worcester Public Healt? Laboratory, and Mr G. Ingram, Consultant Surgeon, Hope Hospital, for their help in the preparation of this paper.

\section{References}

Blaser, M., Cravens, J., Powers, B.W. \& Wong, W.L W (1978) Campylobacter enteritis associated with caning infection. Lancet, ii, 979.

Evans, R.G. \& Dadswell, J.V. (1967) Human vibriosi오 (Letter). British Medical Journal, 3, 240.

Hallett, A.F., Botha, P.L. \& Logan, A. (1977) Isolation of Campylobacter fetus from recent cases of humara vibriosis. Journal of Hygiene, 79, 381.

Simmons, N.A. \& GiBBs, F.J. (1977) Campylobacter enteritis (correspondence). British Medical Journal, 2, 264.

KING, E.O. (1962) The laboratory recognition of Vibrio and a closely related Vibrio isolated from cases of human vibriosis. Annals of the New York Academy of Sciences, 700.

SKIRROw, M.B. (1977) Campylobacter enteritis: a 'news disease. British Medical Journal, $2,9$.

WheEleR, W.E. \& BorChaRs, J. (1961) Vibrionic enteritis i⿱艹 infants. American Journal of Diseases of Children, 101, 60ळ 